\title{
Autologous EBV-Transformed B Lymphoblastoid-Tumor Fusion Cell Vaccine
}

National Cancer Institute

\section{Source}

National Cancer Institute. Autologous EBV-Transformed B Lymphoblastoid-Tumor

Fusion Cell Vaccine. NCI Thesaurus. Code C48377.

A cell-based vaccine composed of autologous tumor cells fused with Epstein-Barr virustransformed B-lymphoblastoid cells. Upon administration, this vaccine may stimulate a cytotoxic T cell response against tumor cells, resulting in tumor cell lysis. ( $\mathrm{NCl05)}$ 